\title{
A prospective 3-year follow-up trial of implantation of two trabecular microbypass stents in open-angle glaucoma
}

This article was published in the following Dove Press journal:

Clinical Ophthalmology

3 November 2015

Number of times this article has been viewed

Eric D Donnenfeld'

Kerry D Solomon ${ }^{2}$

Lilit Voskanyan ${ }^{3}$

David F Chang ${ }^{4}$

Thomas W Samuelson ${ }^{5}$

lqbal Ike K Ahmed ${ }^{6}$

$\mathrm{L}$ Jay Katz ${ }^{7}$

'Ophthalmic Consultants of Long Island, Rockville Centre, NY, ${ }^{2}$ Carolina Eyecare Physicians, Mt Pleasant, SC, USA; ${ }^{3}$ S.V. Malayan Ophthalmology

Centre, Yerevan, Armenia; ${ }^{4}$ Altos Eye Physicians, Los Altos, CA, ${ }^{5}$ Minnesota Eye Consultants, Minneapolis, MN, USA; 'University of Toronto, Toronto, ON, Canada; ${ }^{7}$ Wills Eye Hospital, Jefferson Medical College, Philadelphia, PA, USA
Correspondence: Eric D Donnenfeld Ophthalmic Consultants of Long Island, Ryan Medical Arts Building, 2000 North Village Avenue, Suite 402, Rockville Centre, NY II570, USA

Email ericdonnenfeld@gmail.com
Purpose: To evaluate 3-year safety and intraocular pressure (IOP) following two trabecular microbypass stents in phakic and pseudophakic subjects with open-angle glaucoma (OAG) not controlled on preoperative medication.

Patients and methods: In this prospective pilot study, phakic or pseudophakic subjects with OAG and IOP between $18 \mathrm{mmHg}$ and $30 \mathrm{mmHg}$ on one preoperative topical ocular hypotensive medication underwent medication washout. Thirty-nine qualified subjects with preoperative unmedicated IOP $\geq 22 \mathrm{mmHg}$ and $\leq 38 \mathrm{mmHg}$ received two stents. Postoperative examinations were scheduled at Day 1, Week 1, Months 1, 3, 6, and 12, and semiannually through Month 60 . Ocular hypotensive medication was considered if postoperative IOP exceeded $21 \mathrm{mmHg}$. IOP, medication use, and safety were assessed at each visit. Subject follow-up through Month 36 was completed.

Results: Thirty-six eyes (92.3\%; 95\% confidence interval [CI] 79.1\%, 98.4\%) achieved the primary efficacy end point of Month 12 reduction in IOP $\geq 20 \%$ from baseline (unmedicated IOP) without ocular hypotensive medication. Four subjects required medication during the Month 36 follow-up period. Mean IOP at 36 months for subjects not taking medication was $15.2 \mathrm{mmHg}$. At 36 months, subjects sustained mean IOP decrease of $9.1 \pm 2.7 \mathrm{mmHg}$ (95\% CI $8.0 \mathrm{mmHg}, 10.14 \mathrm{mmHg}$ ), or $37 \%$ IOP reduction, from unmedicated baseline IOP. Compared to preoperative medicated IOP, subjects had mean reduction at Month 36 of $5.5 \pm 2.7 \mathrm{mmHg}(95 \% \mathrm{CI} 4.5 \mathrm{mmHg}, 6.6 \mathrm{mmHg})$, or $26 \%$ reduction. Both measures of IOP reduction were highly significant $(P<0.001)$. Other than one case of early postoperative hyphema that resolved at 1 week, no postoperative adverse events were attributed to stent implantation.

Conclusion: In a pilot study, two trabecular microbypass stents to treat OAG subjects on one preoperative medication provided statistically significant, sustained, and safe reduction of IOP to $\leq 15 \mathrm{mmHg}$ without medication through 36 months.

Keywords: iStent, MIGS, OAG, intraocular pressure, IOP reduction

\section{Introduction}

Microinvasive glaucoma surgery (MIGS) using ab interno trabecular microbypass stents has been shown to be safe and effective for mild-to-moderate glaucoma subjects in conjunction with cataract surgery. These stents bypass the trabecular meshwork, which is thought to be the primary source of resistance to aqueous drainage in most open-angle glaucomas (OAGs), in order to improve outflow through the natural physiologic pathway and reduce intraocular pressure (IOP). The iStent ${ }^{\circledR}$ Trabecular $^{2}$ Micro-Bypass (Glaukos Corp., Laguna Hills, CA, USA) is the first of its kind in the evolving category of MIGS devices and has been shown to offer a safe and effective 
way to lower IOP and medication use in patients with mildto-moderate glaucoma. One small, single-centered prospective, randomized controlled trial ${ }^{1,2}$ and a large, multicentered prospective, randomized study ${ }^{3,4}$ have shown the safety and effectiveness of iStent in conjunction with cataract surgery to reduce IOP and medication burden compared with cataract surgery alone for up to 5 years postoperative; and other studies have corroborated the effects of safe reduction in IOP and medication use vs preoperative parameters for 12 months and 54 months, respectively. 5,6

Use of multiple stents to further increase the outflow has been shown to be viable, both in a theoretical in vitro perfusion model and in clinical experience. Bahler et al's predictive model work showed an increase in the facility of outflow after implantation of multiple stents. ${ }^{7}$ Belovay et al found that implantation of multiple stents during cataract surgery provided mean reduction in IOP to $<15 \mathrm{mmHg}$ with reduction in medications through 1 year postoperative. ${ }^{8}$

Although prior work has established the favorable longterm benefit/risk profile of iStent used in conjunction with cataract surgery, the long-term efficacy of trabecular bypass stent implantation as a sole procedure to manage OAG without the potential pressure lowering effect of cataract surgery has not been established. It is difficult to assess the sole effect of iStent on IOP reduction when implanted during cataract surgery. However, this information is critical for weighing the benefit of surgical therapy in earlier stages of the disease state. Up until the advent of MIGS, trabeculectomy has been recognized as the standard surgical treatment for patients with glaucoma, as have its associated significant complications including hypotony, bleb leaks, cataract progression, choroidal effusion/hemorrhage, and resultant visual deterioration. ${ }^{9}$ Earlier surgical intervention in glaucoma with a safe procedure that restores natural physiologic outflow to achieve target IOP could advance clinical care and significantly reducing drug burden could improve the patient's quality of life. Thus, a series of prospective studies were designed to examine outcomes following stent implantation without cataract surgery in patients at various stages of OAG disease.

This current pilot study, one of the first in a series of studies by the MIGS Study Group, describes outcomes through 3 years postoperatively after two iStent devices were implanted as a stand-alone procedure without concomitant cataract surgery in subjects with OAG not controlled with a single preoperative topical ocular hypotensive medication. This is the first published report of outcomes in our study designed for follow-up through 5 years postoperative.

\section{Materials and methods Subjects}

This prospective, open-label study involved phakic or pseudophakic subjects with OAG (including primary, pigmentary, and pseudoexfoliative). Subjects were required to have a medicated IOP of $\geq 18 \mathrm{mmHg}$ and $\leq 30 \mathrm{mmHg}$ at screening on one topical ocular hypotensive medication. After washout of ocular hypotensive medication (4 weeks for beta-blockers and prostaglandin analogs, 2 weeks for alpha agonists, 5 days for carbonic anhydrase inhibitors), IOP was required to be $\geq 22 \mathrm{mmHg}$ and $\leq 38 \mathrm{mmHg}$ at baseline for inclusion in the trial. Other inclusion criteria included preoperative best-corrected visual acuity (BCVA) of 20/100 or better, visual field defects or nerve abnormality characteristic of glaucoma, willingness to attend follow-up visits for 5 years postoperatively, and informed consent.

The subjects with the following conditions were excluded: narrow angle or secondary glaucoma, aphakia or pseudophakia with anterior chamber intraocular lenses, prior stent implantation, peripheral anterior synechiae, prior argon laser trabeculoplasty, prior iridectomy or laser iridotomy, active corneal inflammation or edema, prior corneal surgery, corneal opacities/disorders inhibiting visualization of the nasal angle, elevated episcleral venous pressure, functionally significant visual field loss, and chronic or active ocular inflammation.

\section{Study group and protocol registration}

The MIGS Study Group was formed in order to evaluate iStent technology as titratable therapy in a series of prospective studies in over 2,000 patients. For many of the surgeons, this was their first experience implanting a MIGS device. The study group comprises visiting surgeons and one staff surgeon at one investigational site (S.V. Malayan Ophthalmology Centre, Yerevan, Armenia).

The study protocol received Ethics Committee approval, and informed consent was obtained from patients for study participation. Methods of data collection were in accordance with the Declaration of Helsinki (2008). The study was registered and assigned clinical trial registration number NCT1252862 (Clinicaltrials.gov).

\section{iStent, surgical technique, examinations, and follow-up}

The iStent is a heparin-coated, gamma-sterilized device made from titanium. The one-piece device is $\sim 1.0 \mathrm{~mm}$ in length, $\sim 0.33 \mathrm{~mm}$ in height, with a snorkel bore diameter of $120 \mu \mathrm{m}$. Left-flow stents and right-flow stents are 
available. These stents are identical except that the "foot" of each faces opposite directions to facilitate nasal stent placement and to optimize distal flow through collector channels. When implanted, the snorkel is located in the anterior chamber, and the open half-pipe lumen (foot) is located in the canal of Schlemm. A stainless steel singleuse inserter containing the preloaded stent is used for stent implantation.

Using the technique described by Samuelson et al and Spiegel et al, ${ }^{1,10}$ two iStent devices were implanted through the trabecular meshwork into Schlemm's canal at the nasal position, separated by two clock hours and facing opposite directions (Figure 1). Viscoelastic was removed via manual irrigation with balanced salt solution. A postoperative regimen of topical anti-inflammatory and anti-infective medications was followed for 4 weeks. Topical ocular hypotensive medication was considered for subjects in whom IOP increased to $>21 \mathrm{mmHg}$.

Following the informed consent process, the screening examination included slit-lamp biomicroscopy, indirect ophthalmoscopy, measurement of IOP, BCVA (via Early Treatment of Diabetic Retinopathy Study system), visual field (automated threshold visual field via Humphrey 24-2, Swedish Interactive Thresholding Algorithm (SITA) Standard), and pachymetry. After washout of ocular hypotensive medications according to the medication class, subjects underwent baseline examination for evaluation of IOP. Those subjects whose IOP values were within the required range were then scheduled to undergo surgery. Follow-up visits were scheduled at Day 1, Week 1, Months 1, 3, 6, and 12, followed by visits every 6 months for a total of 5 years and consisted of examination parameters similar to the set of preoperative measurements. Glaucoma-trained ophthalmologists at the eye hospital performed preoperative and postoperative IOP measurements at

A

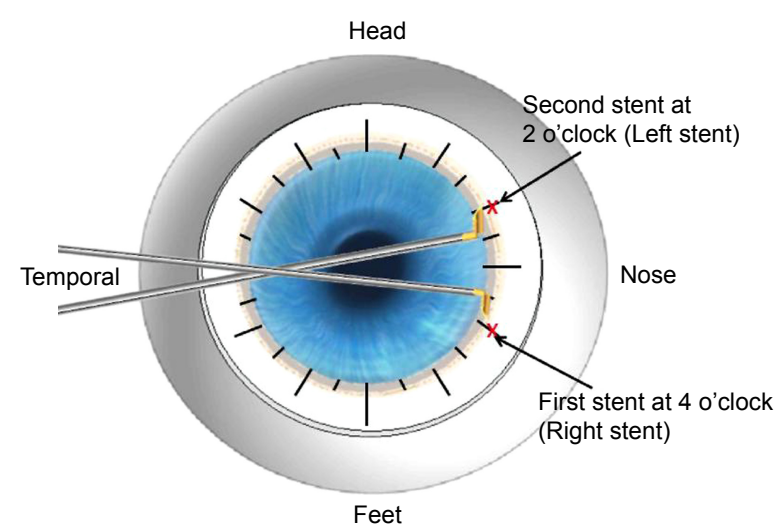

$9 \mathrm{am} \pm 1$ hour. In addition, the medical monitor of the trial (LJK) evaluated the IOP of a sample of these patients as well.

\section{Study end points and statistical analysis}

Two efficacy end points were defined in the study protocol. Month 12 IOP reduction $\geq 20 \%$ without medication vs baseline unmedicated IOP comprised the primary end point, and Month 12 IOP $\leq 18 \mathrm{mmHg}$ without medication was the secondary end point. Subjects were considered responders if they met all criteria for the end points, including no use of ocular hypotensive medications at the Month 12 examination and no secondary surgical intervention to reposition or remove stents or other procedure that could affect IOP before the Month 12 examination. We assessed additional efficacy at Month 36, including mean unmedicated IOP, mean change in IOP from preoperative to Month 36 unmedicated IOP, and medication use. Safety analyses through Month 36 involved assessment of adverse events, BCVA, slit lamp findings, and pachymetry.

The per protocol analysis population included qualified subjects who underwent implantation of two stents. Exact 95\% confidence intervals (CIs) based on a binomial distribution were calculated for responder rates of proportional analyses. For continuous variables, such as mean IOP, 95\% CIs were calculated using the $t$-distribution. Change in IOP from preoperative to Month 36 was assessed via paired $t$-tests. Statistical analysis software was used (PC-SAS software, Version 9.3; SAS Institute Inc., Cary, NC, USA).

\section{Results}

\section{Subject disposition and demographics}

A total of 94 subjects were screened for study participation. Eighteen subjects did not meet screening criteria, and 36 subjects did not meet baseline criteria (eg, did not

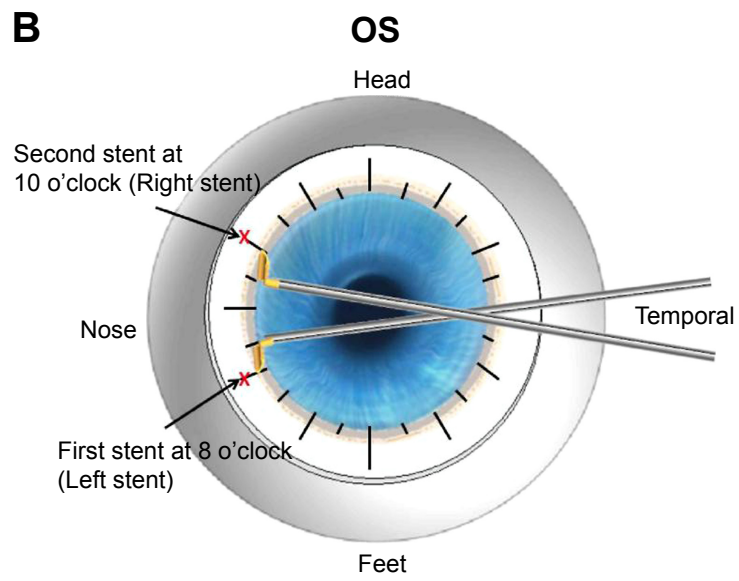

Figure I Example of iStent locations.

Notes: (A) OD (right eye): left stent at 2 o'clock, right stent at 4 o'clock. (B) OS (left eye): left stent at 8 o'clock, right stent at I0 o'clock. 
complete medication washout, withdrew consent, IOP too high after medication washout). The remaining 40 qualified subjects underwent surgery. In addition, two subjects who were taking combination medications preoperatively underwent surgery. Because their preoperative medication burden was later categorized as two medications, these two subjects were classified as protocol deviations. All 42 surgeries were performed at a single investigational site (S.V. Malayan Ophthalmology Centre, Yerevan, Armenia), and surgeries were performed by six surgeons, including the local surgeon and five visiting surgeons from the USA and Canada.

Of the 42 subjects who underwent surgery, 39 subjects were included in the per protocol analysis population (Figure 2). Of the three subjects excluded from the per protocol population, two subjects with preoperative combination medications did not meet the screening criteria (discussed earlier) and one subject underwent implantation of only one stent due to unintended subject movement during surgery. The per protocol analysis population also includes data from five subjects who presented with preoperative BCVA of $20 / 200$ (worse than the inclusion criteria of $20 / 100$ or better), four of whom had eccentric vision, who were granted protocol waivers by the sponsor and allowed to participate in the study. All 39 per protocol subjects presented with follow-up through 24 months. Nine subjects were exited after the Month 24 examination and before the Month 36 examination. Two subjects had died, two subjects had moved out of the country, and five subjects were lost to follow-up.

Demographics are shown in Table 1. The mean age at enrollment was $66.7 \pm 10.0$ years. Twenty-two subjects (56.4\%) were male. Subjects were primarily phakic $(89.7 \%)$ with brown irides $(94.9 \%)$. The mean visual field (mean deviation) was $-4.95 \pm 2.52 \mathrm{~dB}$. The mean cup:disk (C:D) ratio was $0.7 \pm 0.2$, and eleven eyes $(28.2 \%)$ had $\mathrm{C}: \mathrm{D}$ ratios of worse than 0.8 . Subjects presented with mean pachymetry values of $535 \pm 32.7 \mu \mathrm{m}$. Mean medicated IOP at screening was $20.6 \pm 2.0 \mathrm{mmHg}$, with most subjects (56.4\%) taking beta-blockers preoperatively. Mean IOP following medication washout was $24.1 \pm 1.4 \mathrm{mmHg}$.

\section{Intraocular pressure}

The primary efficacy end point (IOP reduction $\geq 20 \%$ from baseline to 12 months without medication) and the

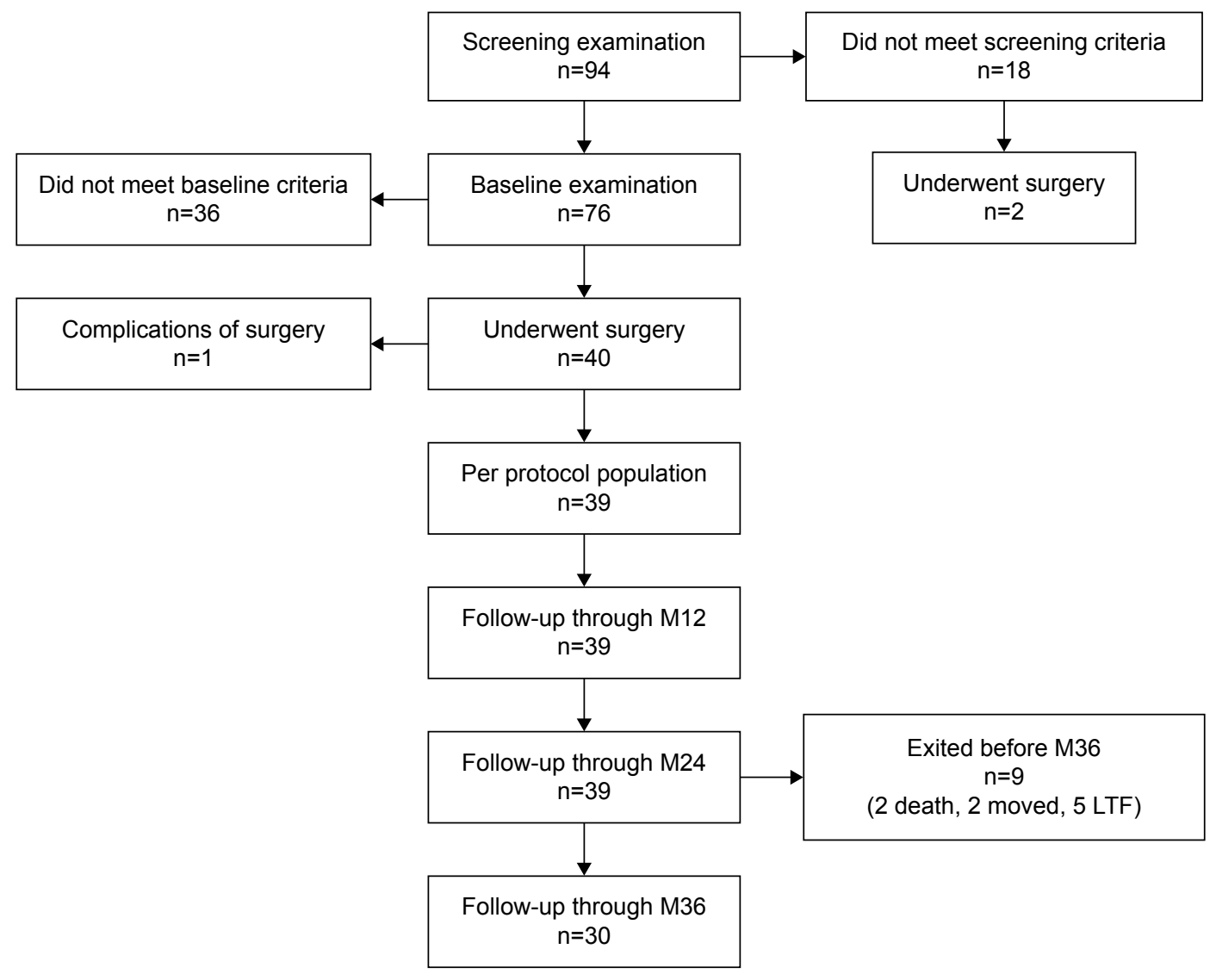

Figure 2 Subject flowchart.

Abbreviations: LTF, lost to follow up; M, month. 
Table I Demographic and preoperative characteristics

\begin{tabular}{|c|c|}
\hline Variable & Statistic \\
\hline $\mathrm{N}$ & 39 \\
\hline \multicolumn{2}{|l|}{ Mean age (years) } \\
\hline Mean \pm SD & $66.7 \pm 10.0$ \\
\hline Range & $50-90$ \\
\hline \multicolumn{2}{|l|}{ Sex } \\
\hline Male & $22(56.4 \%)$ \\
\hline Female & $17(43.6 \%)$ \\
\hline \multicolumn{2}{|l|}{ Race/ethnicity } \\
\hline White & $39(100.0 \%)$ \\
\hline \multicolumn{2}{|l|}{ Lens status } \\
\hline Phakic & 35 (89.7\%) \\
\hline Pseudophakic & $4(10.3 \%)$ \\
\hline \multicolumn{2}{|l|}{ Eye } \\
\hline OD & $16(41.0 \%)$ \\
\hline OS & $23(59.0 \%)$ \\
\hline \multicolumn{2}{|l|}{ Iris color } \\
\hline Brown & 37 (94.9\%) \\
\hline Blue & $2(5.1 \%)$ \\
\hline \multicolumn{2}{|l|}{ Mean medicated IOP $(\mathrm{mmHg})$} \\
\hline Mean \pm SD & $20.6 \pm 2.0$ \\
\hline \multicolumn{2}{|l|}{ Medications } \\
\hline Beta-blocker & $22(56.4 \%)$ \\
\hline Carbonic anhydrase inhibitor & $10(25.6 \%)$ \\
\hline Prostaglandin analog & $6(15.4 \%)$ \\
\hline Alpha agonist & $\mathrm{I}(2.6 \%)$ \\
\hline \multicolumn{2}{|l|}{ Visual field $(\mathrm{dB})$} \\
\hline Mean deviation, mean \pm SD & $-4.95 \pm 2.52$ \\
\hline Pattern standard deviation, mean \pm SD & $3.05 \pm 2.15$ \\
\hline \multicolumn{2}{|l|}{ C:D ratio } \\
\hline Mean \pm SD & $0.7 \pm 0.2$ \\
\hline Range & $0.4-0.95$ \\
\hline \multicolumn{2}{|l|}{ Mean pachymetry $(\mu \mathrm{m})$} \\
\hline Mean \pm SD & $535 \pm 32.7$ \\
\hline \multicolumn{2}{|l|}{ Mean postwashout IOP (mmHg) } \\
\hline Mean \pm SD & $24.1 \pm 1.4$ \\
\hline
\end{tabular}

Note: C:D ratio denotes cup:disk ratio.

Abbreviations: SD, standard deviation; IOP, intraocular pressure; OD, right eye; OS, left eye.

secondary end point (IOP $\leq 18 \mathrm{mmHg}$ at 12 months without medication) were each achieved by $92.3 \%$ of subjects $(n=36$; 95\% CI 79.1\%, 98.4\%; Figure 3). The mean reduction in IOP from baseline was $44 \%$. Only the three subjects taking ocular hypotensive medications at 12 months were considered nonresponders for each of the efficacy end points. No subject underwent secondary surgical intervention prior to the Month 12 examination that could affect IOP. Most subjects maintained these target IOP thresholds through Month 36, with IOP reduction $\geq 20 \%$ achieved by $86.2 \%$ ( $n=25 ; 95 \%$ CI $68.3 \%, 96.1 \%$ ) and IOP $\leq 18 \mathrm{mmHg}$ achieved by $89.7 \%$ $(n=26 ; 95 \%$ CI $72.6 \%, 97.8 \%)$.

Mean IOP for subjects not using ocular hypotensive medication was $13.5 \pm 1.8 \mathrm{mmHg}$ at Month 12 (95\% CI 12.9 $\mathrm{mmHg}, 14.1 \mathrm{mmHg}), 13.5 \pm 2.1 \mathrm{mmHg}$ at Month $24(95 \%$

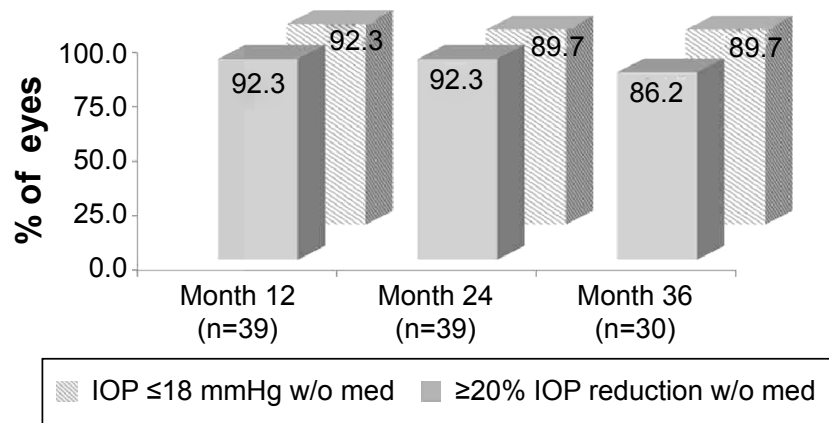

Figure 3 Proportional analyses of IOP outcomes at annual examinations through Month 36, per protocol population.

Notes: IOP values were excluded after secondary surgeries. One subject had cataract surgery after M24 and was excluded from the M36 analyses.

Abbreviations: IOP, intraocular pressure; w/o, without; med, medication; M, month.

CI $12.8 \mathrm{mmHg}, 14.2 \mathrm{mmHg})$, and $15.2 \pm 2.1 \mathrm{mmHg}(95 \%$ CI $14.3 \mathrm{mmHg}, 16.0 \mathrm{mmHg}$ ) at Month 36. In the consistent cohort of subjects followed through Month 36 and not using medications, mean IOP values were similar to the set of all eyes. Mean Month 36 IOP decrease from unmedicated baseline IOP was $9.1 \pm 2.7 \mathrm{mmHg}(95 \%$ CI $8.0 \mathrm{mmHg}$, $10.14 \mathrm{mmHg}$ ). Compared to preoperative medicated IOP, these subjects experienced a mean IOP reduction of $5.5 \pm 2.7 \mathrm{mmHg}(95 \%$ CI $4.5 \mathrm{mmHg}, 6.6 \mathrm{mmHg})$ at their Month 36 examination. Both the mean reduction in Month 36 IOP from unmedicated baseline and the mean Month 36 IOP decrease from subjects' medicated screening IOP were highly significant $(P<0.001$ in each case). Figure 4 shows boxplots of IOP over time for the consistent cohort.

\section{Ocular hypotensive medications}

The vast majority of eyes (35/39 or $89.7 \%$ ) did not require ocular hypotensive medications following two stent implantations. Of the four subjects treated with postoperative medication, medication was initiated at 1 week postoperative in

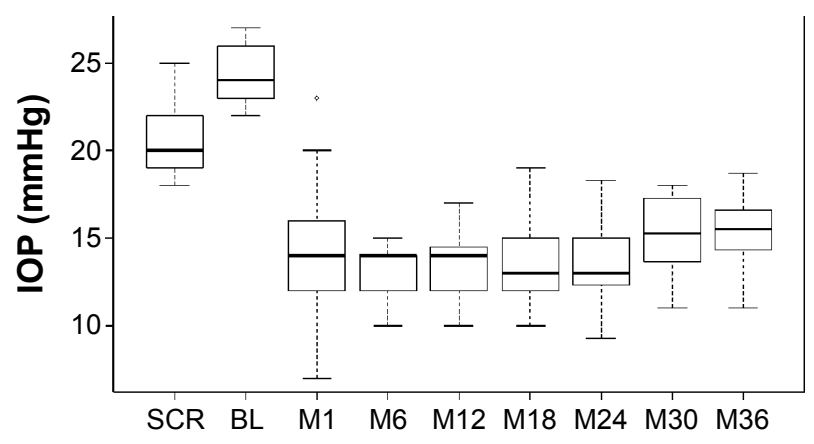

Figure 4 IOP over time, consistent cohort through Month $36(n=30)$.

Notes: IOP values were excluded for eyes on postoperative medication and after secondary surgeries. One subject had cataract surgery after M24 and was excluded from subsequent statistics.

Abbreviations: IOP, intraocular pressure; M, month; SCR, screening; BL, baseline. 
Table 2 BCVA by visit, per protocol population

\begin{tabular}{|c|c|c|c|c|c|c|c|}
\hline & PRE & M6 & MI2 & MI8 & M24 & M30 & M36 \\
\hline $\mathrm{N}$ & 39 & 37 & 38 & 39 & 39 & 30 & 30 \\
\hline $20 / 40$ or better & $26(66.7 \%)$ & $26(70.3 \%)$ & 28 (73.7\%) & 28 (7I.8\%) & 28 (7I.8\%) & $23(76.7 \%)$ & $23(76.7 \%)$ \\
\hline $20 / 100$ or better & 34 (87.2\%) & 32 (86.5\%) & 33 (86.8\%) & 34 (87.2\%) & 34 (87.2\%) & $26(86.7 \%)$ & 27 (90.0\%) \\
\hline $20 / 200$ or better & $39(100.0 \%)$ & $37(100.0 \%)$ & 38 (100.0\%) & $39(100.0 \%)$ & $39(100.0 \%)$ & $30(100.0 \%)$ & $30(100.0 \%)$ \\
\hline
\end{tabular}

Abbreviations: BCVA, best-corrected visual acuity; $M$, month; PRE, preoperative.

two subjects and at 1 month postoperative in two subjects. Medication use was discontinued in one subject at the Month 3 examination and in one subject at the Month 24 examination. Thus, three subjects were on medication at the Month 12 and Month 24 examinations, and two subjects were on medications at the Month 36 examination. Month 36 IOP for these four subjects ranged from $13 \mathrm{mmHg}$ to $15.7 \mathrm{mmHg}$.

\section{Best-corrected visual acuity, slit lamp, pachymetry}

The proportion of eyes with BCVA of 20/40 or better was $66.7 \%$ at the screening examination, $73.7 \%$ at 12 months postoperative, $71.8 \%$ at 24 months, and $76.7 \%$ at 36 months (Table 2). Three eyes experienced BCVA loss of $\geq 1$ line from preoperative BCVA due to progression of preexisting cataract not related to stent implantation. All three subjects had bilateral cataracts. One of these subjects underwent cataract surgery and reported with 20/20 BCVA at their Month 36 examination. The other two subjects had last reported BCVA of 20/50 and 20/40. No abnormal slit lamp findings were noted after the 1-week postoperative visit. C:D ratio was stable over time (Table 3) as was visual field (Table 4). Mean central corneal thickness was stable over time $(535.0 \pm 32.7 \mu \mathrm{m}$ at screening and $538.7 \pm 33.8 \mu \mathrm{m}$ at 36 months).

\section{Adverse events and secondary surgical intervention}

Ten adverse events were reported in eight eyes in the per protocol population (Table 5). As stated earlier, BCVA loss of $\geq 1$ line due to progression of existing cataract was reported in three subjects, with subsequent cataract surgery performed

Table 3 C:D ratio by visit, per protocol population

\begin{tabular}{lllll}
\hline & PRE & MI2 & M24 & M36 \\
\hline $\mathrm{N}$ & 39 & 39 & 39 & 30 \\
$\leq 0.5$ & $6(15.4 \%)$ & $6(15.4 \%)$ & $6(15.4 \%)$ & $5(16.7 \%)$ \\
$>0.5$ and $\leq 0.8$ & $22(56.4 \%)$ & $22(56.4 \%)$ & $22(56.4 \%)$ & $18(60.0 \%)$ \\
$>0.8$ & II (28.2\%) & II (28.2\%) & II (28.2\%) & $7(23.3 \%)$ \\
\hline
\end{tabular}

Note: $C: D$ ratio $=$ cup:disk ratio

Abbreviations: M, month; PRE, preoperative. in one subject. Two subjects died during the postoperative follow-up. One subject experienced hyphema that filled the anterior chamber at 2 weeks postoperative, requiring surgical irrigation of the anterior chamber. At the time of the event, IOP was $30 \mathrm{mmHg}$ and BCVA was hand movement. The vision improved after clearing the hyphema, and IOP decreased after administration of topical ocular hypotensive medication. Medication was discontinued at Month 3, at which time IOP was $11 \mathrm{mmHg}$. The subject presented with initial cataract over the postoperative period that was deemed unrelated to the study treatment. At Month 36, this subject's IOP was $13 \mathrm{mmHg}$ on no medication with BCVA $=20 / 25$. Proliferative diabetic retinopathy was reported in one subject, and scar from age-related macular degeneration was reported in one subject.

\section{Discussion}

Because of the transient IOP reduction associated with phacoemulsification alone, the efficacy of iStent implantation is more difficult to gauge when combined with cataract surgery. This is the first MIGS study to evaluate long-term outcomes of implantation of two iStent devices as a stand-alone treatment for patients with OAG. The study involved multiple surgeons, thereby yielding some external validation of the safety of the surgical technique. The data presented in this publication span a 3-year postoperative period. The high level of subject accountability, including $100 \%$ accountability through 2 years and only five subjects lost to follow-up at 3 years, adds to the strength of the evidence from this small study.

Table 4 Visual field, ${ }^{a}$ per protocol population

\begin{tabular}{|c|c|c|c|c|}
\hline & PRE & MI2 & M24 & M36 \\
\hline $\mathrm{N}$ & 35 & 35 & 35 & 27 \\
\hline \multicolumn{5}{|c|}{ Mean deviation } \\
\hline Mean $(\mathrm{dB})$ & -4.95 & -4.16 & -4.00 & -3.97 \\
\hline $\mathrm{SD}(\mathrm{dB})$ & 2.52 & 2.66 & 3.14 & 2.31 \\
\hline \multicolumn{5}{|c|}{ Pattern standard deviation } \\
\hline Mean $(\mathrm{dB})$ & 3.05 & 2.82 & 2.96 & 2.37 \\
\hline $\mathrm{SD}(\mathrm{dB})$ & 2.15 & 2.36 & 2.22 & 1.63 \\
\hline
\end{tabular}

Notes: ${ }^{A}$ Four subjects with eccentric VA did not have visual field measurements. One of these subjects did not have a Month 36 examination (died before examination). Abbreviations: SD, standard deviation; M, month; PRE, preoperative; VA, visual acuity. 
Table 5 Adverse events, per protocol population

\begin{tabular}{ll}
\hline Event & $\mathbf{N}=\mathbf{3 9 , \mathbf { n } ( \% )}$ \\
\hline BCVA loss due to progression of preexisting cataract & $3(7.7)$ \\
Death & $2(5.1)$ \\
Hyphema & $\mathrm{I}(2.6)$ \\
Initial cataract & $\mathrm{I}(2.6)$ \\
Proliferative diabetic retinopathy & $\mathrm{I}(2.6)$ \\
Scar from age-related macular degeneration & $\mathrm{I}(2.6)$ \\
Secondary surgical intervention (cataract surgery) & $\mathrm{I}(2.6)$ \\
Total adverse events & $\mathrm{I}(25.6)$ \\
Total subjects with adverse events & $8(20.5)$ \\
\hline
\end{tabular}

Abbreviation: BCVA, best-corrected visual acuity.

This prospective pilot study showed that phakic and pseudophakic subjects with OAG can safely be implanted with two iStent devices. No device related adverse events were observed, and no subjects experienced hypotony. Adverse events mostly involved BCVA loss due to progression of preexisting cataract. In patients under such a health care system, surgery is reserved for advanced vision loss. With the exception of one procedure-related early postoperative hyphema that resolved at 1 week after irrigation and without further sequelae, no adverse events were considered related to iStent implantation.

Subjects experienced reduction in IOP through 3 years postoperative from their unmedicated baseline IOP that was both clinically significant (37\%) and statistically significant $(P<0.001)$. Of even greater significance is that subjects' IOP was $26 \%$ lower $(P<0.001)$ without medications 3 years after receiving iStents than with medications before surgery. Many glaucoma patients will require multiple medications for their disease. Although adding a second medication typically reduces IOP by an additional 2.5-3.9 mmHg, ${ }^{11,12}$ the incidence of medication nonadherence has been shown to range from $30 \%$ to $80 \%{ }^{13,14}$ Because the mean IOP in our study was $5.5 \mathrm{mmHg}$ lower 3 years after surgery compared to preoperative IOP on one medication, it is likely that these subjects' IOP would have been higher had they been prescribed multiple medications instead of receiving two stents. Most subjects experienced significant IOP reduction without the need for medication; only two subjects were taking ocular hypotensive medication at their Month 36 examination. A key advantage of ab interno glaucoma implants over medications is reduced dependence on patient compliance, and this might theoretically improve long-term outcomes. Thus, the well-known limitations of medical therapy administration, persistence, and adherence are avoided with an implantable glaucoma device that provides continuous therapy independent of patient or physician involvement.
Numerous studies have shown IOP and medication reduction after implantation of a single stent in conjunction with cataract surgery in patients with mild-to-moderate disease on one to three medications. The work by Samuelson et al, Craven et al, and Fea demonstrated IOP reduction of $\leq 18 \mathrm{mmHg}$ with reduced medication burden through 1 year postoperative. ${ }^{1-3}$ In the US pivotal trial, mean IOP at 1 year was $17.0 \pm 2.8 \mathrm{mmHg}$ in the iStent group on an average number of $0.2 \pm 0.6$ ocular hypotensive medications. ${ }^{1}$ Although mean IOP in the stent group and control group at 1 year was similar, the mean number of medications required to control IOP was significantly lower in the stent group $(0.2 \pm 0.6$ vs $0.4 \pm 0.7 ; P=0.011) .^{2}$ Furthermore, Samuelson et al showed $66 \%$ of subjects implanted with one iStent in conjunction with cataract surgery achieved an IOP reduction of $\geq 20 \% 1$ year after surgery without the need for ocular hypotensive medication. ${ }^{1}$ After 2 years postoperative, $61 \%$ of subjects from this study still reported IOP reduction of $\geq 20 \%$ from baseline. ${ }^{2}$ A separate study showed that eyes implanted with iStent during cataract surgery reported with a mean IOP decrease of $16.33 \% 3$ years after surgery and $47 \%$ did not require ocular hypotensive medication. ${ }^{6}$ In our study, $92.3 \%$ of subjects had IOP reduction $>20 \%$, without medication at 1 year, and $66.7 \%$ experienced $40 \%$ reduction.

Belovay et al showed that implantation of two or three iStent devices in conjunction with cataract surgery in patients with moderate-to-severe disease on two to three medications resulted in IOP reduction to a mean IOP of $14.3 \mathrm{mmHg} 1$ year postoperatively. ${ }^{8}$ These findings are in accord with initial work established by Bahler et al that showed significant increased outflow facility and reduced IOP after implantation of a single stent and further increase in outflow facility and reduced IOP when multiple stents were implanted. ${ }^{7}$

More recent work by Ahmed et al demonstrated safety and effectiveness through 18 months following treatment with two trabecular bypass stents and one presumptive postoperative prostaglandin in patients with OAG not controlled on two preoperative medications. ${ }^{15}$ In their study, patients achieved IOP reduction of $20 \%$ with reduction of one medication from their preoperative status, with IOP $\leq 14 \mathrm{mmHg}$ through 1.5 years. Our current work focused on eyes in a slightly earlier stage of disease. In our study, IOP in OAG eyes not controlled on one preoperative medication was managed to a mean of $15.2 \mathrm{mmHg}$ at 3 years postoperatively without ocular hypotensive medication. While the various studies are not directly comparable, they do show that single and multiple iStent devices are capable of achieving, and 
sustaining through several years, target IOPs for various stages of disease. Multiple iStent devices may be a viable option for patients who need additional IOP reduction in order to delay the progression of glaucoma.

Several limitations of this study were identified. Because of the small number of pseudophakic subjects, outcomes were not stratified by preoperative lens status. A control group was not included, and the study design was open label. All subjects were Caucasian, and all subject evaluations were performed at a single site. Therefore, it is not possible to assess study site effects or measurement variability across study centers. Measurements of IOP at each examination were not recorded at multiple time points. The preoperative status of the crystalline lens was not graded. This population had not undergone previous incisional surgery, and most subjects were on a preoperative regimen of beta-blockers. The significant IOP reduction observed in this study may be greater than IOP reduction in eyes with more advanced glaucoma and/or in which the distal outflow system is less functional. However, the long-term data collected in a stable cohort over the 3-year period offer valuable insight about this treatment method. Continued IOP monitoring of this patient cohort is a part of an ongoing study. We also welcome larger, multicenter controlled studies to further corroborate our findings.

Our study confirmed that long-term IOP reduction to $15 \mathrm{mmHg}$ is possible after implantation of multiple iStent devices without cataract surgery, without postoperative medication burden, and without significant postoperative adverse effects. Management of chronic OAG with multiple medications is often associated with poor compliance and with local or systemic side effects. A safe and effective implant procedure that provides a clinically and statistically significant IOP reduction in mild-to-moderate OAG may be a preferable alternative for some patients.

\section{Acknowledgments}

Glaukos Corporation provided study devices, sponsorship for performing this study and conducting data analyses, editorial assistance in the preparation of this manuscript, and payment of the article processing charges.

\section{Disclosure}

Drs Donnenfeld, Solomon, Chang, Samuelson, Ahmed, and Katz received nonfinancial support for their work as investigators in this study. Drs Voskanyan, Chang, and Samuelson received financial support from Glaukos for their work as investigators in this study. Dr Katz received financial support from Glaukos for his work as a medical monitor for this study. Drs Donnenfeld, Solomon, Samuelson, Ahmed, and Katz also received nonstudy financial support from Glaukos. Dr Katz served as the medical monitor for this study. All authors are consultants of Glaukos. The authors report no other conflicts of interest in this work.

\section{References}

1. Samuelson TW, Katz LJ, Wells JM, Duh Y-J, Giamporcaro JE; US iStent Study Group. Randomized evaluation of the trabecular microbypass stent with phacoemulsification in patients with glaucoma and cataract. Ophthalmology. 2011;118:459-467.

2. Craven ER, Katz LJ, Wells JW, Giamporcaro JE; iStent Study Group. Cataract surgery with trabecular micro-bypass stent implantation in patients with mild-to-moderate open-angle glaucoma and cataract: two-year follow-up. J Cataract Refract Surg. 2012;38:1339-1345.

3. Fea AM. Phacoemulsification versus phacoemulsification with microbypass stent implantation in primary open-angle glaucoma. J Cataract Refract Surg. 2010;36:407-412.

4. Fea AM, Pignata G, Bartoli E, et al. Prospective, randomized, doublemasked trial of trabecular bypass stent and cataract surgery vs. cataract surgery alone in primary OAG: long-term data. Presented at: 2012 European Society of Cataract and Refractive Surgeons; September, 2012; Milan, Italy.

5. Spiegel D, Wetzel W, Neuhann T, et al. Coexistent primary openangle glaucoma and cataract: interim analysis of a trabecular microbypass stent and concurrent cataract surgery. Eur J Ophthalmol. 2009; 19:393-399.

6. Arriola-Villalobos P, Martínez-de-la-Casa JM, Díaz-Valle D, Fernández-Pérez C, García-Sánchez J, García-Feijoó J. Combined iStent trabecular micro-bypass stent implantation and phacoemulsification for coexistent open-angle glaucoma and cataract: a long-term study. Br J Ophthalmol. 2012;96(5):645-649.

7. Bahler C, Smedley G, Zhou J, Johnson D. Trabecular bypass stents decrease intraocular pressure in cultured human anterior segments. Am J Ophthal. 2004;138:988-994.

8. Belovay GW, Naqi A, Chan BJ, Rateb M, Ahmed II. Using multiple trabecular micro-bypass stents in cataract patients to treat open-angle glaucoma. J Cataract Refract Surg. 2012;38(11):1911-1917.

9. Rulli E, Biagioli E, Riva I, et al. Efficacy and safety of trabeculectomy vs nonpenetrating surgical procedures. A systematic review and metaanalysis. JAMA Ophthalmol. 2013;131(12):1573-1582.

10. Spiegel D, Garcia-Feijoo J, Garcia-Sanchez J, Lamielle H. Coexistent primary open-angle glaucoma and cataract: preliminary analysis of treatment by cataract surgery and the iStent trabecular micro-bypass stent. Adv Ther. 2008;25:453-464.

11. O'Connor DJ, Martone JF, Mead A. Additive intraocular pressure lowering effect of various medications with latanoprost. Am J Ophthalmol. 2002;133:836-837.

12. Reis R, Queiroz CF, Santos LC, Avila MP, Magacho L. A randomized, investigator-masked, 4-week study comparing timolol maleate $0.5 \%$, brinzolamide $1 \%$, and brimonidine tartrate $0.2 \%$ as adjunctive therapies to travoprost $0.004 \%$ in adults with primary open-angle glaucoma or ocular hypertension. Clin Ther. 2006;28:552-559.

13. OlthoffCM, Schouten JS, van de Borne BW, Webers CA. Noncompliance with ocular hypotensive treatment in patients with glaucoma or ocular hypertension an evidence-based review. Ophthalmology. 2005; 112:953-961.

14. Schwartz GF, Quigley HA. Adherence and persistence with glaucoma therapy. Surv Ophthalmol. 2008;53(suppl 1):S57-S68.

15. Ahmed II, Katz LJ, Chang DF, et al. Prospective evaluation of microinvasive glaucoma surgery with trabecular microbypass stents and prostaglandin in open-angle glaucoma. J Cataract Refract Surg. 2014; 40:1295-1300. 
Clinical Ophthalmology

\section{Publish your work in this journal}

Clinical Ophthalmology is an international, peer-reviewed journal covering all subspecialties within ophthalmology. Key topics include: Optometry; Visual science; Pharmacology and drug therapy in eye diseases; Basic Sciences; Primary and Secondary eye care; Patien Safety and Quality of Care Improvements. This journal is indexed on

Submit your manuscript here: http://www.dovepress.com/clinical-ophthalmology-journal

PubMed Central and CAS, and is the official journal of The Society of Clinical Ophthalmology (SCO). The manuscript management system is completely online and includes a very quick and fair peer-review system, which is all easy to use. Visit http://www.dovepress.com/ testimonials.php to read real quotes from published authors. 